\title{
DER STRATEGISCHE AUSBAU DER FORSCHUNG AN ÖSTERREICHISCHEN FACHHOCHSCHULEN
}

\author{
GÜNTHER R. BURKERT, BARBARA HELLER-SCHUH, KARL-HEINZ LEITNER AND GEORG ZAHRADNIK
}

\section{EINLEITUNG}

$\mathrm{F}$ Torschung an Fachhochschulen und hier insbesondere verwertungsorientierte und wirtschaftsnahe Forschung gewinnt in den letzten Jahren zunehmend an Bedeutung. Fachhochschulen haben dabei in der Vergangenheit unterschiedliche öffentliche und private Finanzierungsquellen sowohl auf nationaler als auch europäischer Ebene erschlossen. So wurden beispielsweise durch spezifische nationale Förderprogramme wie das FFG-Programm COIN Cooperation \& Innovation und dessen Vorläuferprogramm FHplus aber auch durch Förderungen von Seiten der Länder Möglichkeiten und Anreize für den gezielten Ausbau von Forschungsinfrastrukturen und zur Stärkung der Humanressourcen in enger Abstimmung zwischen Forschungs- und Lehraktivitäten gelegt. Einige Studien dokumentieren den starken Anstieg der Forschungsaktivitäten und kommen zum Schluss, dass die Fachhochschulen nicht nur eine wichtige Ausbildungsfunktion wahrnehmen, sondern mittlerweile ein wichtiger Akteur im Forschungs- und Innovationssystem sind. ${ }^{1}$

Für den gezielten Ausbau von Forschungsaktivitäten hat ein Teil der Fachhochschulen explizite Forschungsstrategien entwickelt. Die Entwicklung derartiger Strategien erfolgt dabei häufig in enger Abstimmung mit den Stakeholdern der Fachhochschulen. ${ }^{2}$ Insbesondere Länder haben sich in diesen Prozess eingebracht, um das Potential der Fachhochschulen für ihre spezifischen Anforderungen nutzbar zu machen. Das Wachstum der Fachhochschulen stellt diese allerdings vor strategische Herausforderungen in Bezug auf die Ausrichtung der Forschungsaktivitäten in Abstimmung mit den Ausbildungs- und Lehraktivitäten und der Frage, ob eine Diversifizierung oder Spezialisierung verfolgt werden soll. Da die thematische Ausrichtung von Fachhochschulen zugleich von den Finanzierungsmöglichkeiten und der Nachfragestruktur im regionalen Umfeld geprägt ist, orientieren sich Fachhochschulen vornehmlich an den Erfordernissen der Lehre und Studienprogramme, welche sich wiederum an den Bedürnissen der Wirtschaft und Gesellschaft ausrichten.

Fachhochschulen müssen sich beim Ausbau ihrer Forschungsaktivitäten auch gegenüber Universitäten und anderen Forschungseinrichtungen positionieren und gezielt Kooperationspartner suchen. Der Österreichische Wissenschaftsrat argumentiert in diesem Zusammenhang etwa, dass gelungene Forschungskooperationen zwischen Universitäten und
Fachhochschulen mit passenden Schwerpunkten eine besondere Rolle spielen, weil diese neben der Verknüpfung von angewandter Forschung und Grundlagenforschung, auch der Akademisierung des Personals an den Fachhochschulen und der Förderung des wissenschaftlichen Nachwuchses dienen. ${ }^{3}$ Wie im Kontext des Projekts "Zukunft Hochschule“ formuliert wurde, sollte die Leitlinie dabei sein, sich voneinander zu unterscheiden und zu kooperieren, anstelle sich aneinander anzugleichen und zu konkurrieren. ${ }^{4}$

Empirische Befunde über die Entwicklung der Forschungsaktivitäten illustrieren zugleich, dass diese im Fachhochschulsektor heterogen verteilt sind und eine Konzentration auf einige forschungsstarke Fachhochschulen mit spezifischen Ausbildungsschwerpunkten beobachtet werden kann. Wenngleich Studien die Entwicklung und den starken Ausbau der Forschung an Fachhochschulen dokumentieren, existieren bislang keine systematischen Befunde über die thematische Ausrichtung im Spannungsfeld zwischen Fokussierung und Diversifizierung und die Bedeutung unterschiedlicher Finanzierungsquellen. Der vorliegende Beitrag kombiniert dabei erstmals Daten der FH BIS Verordnung, von Forschungsförderungsfonds und aus den Wissensbilanzen der Universitäten, um die Forschungsaktivitäten thematisch zu verorten und (potentielle) Kooperationspartner zu identifizieren.

Im Beitrag werden zunächst die verwendeten Daten und Methoden beschrieben, sodann werden die Ergebnisse zur Finanzierung, Spezialisierung und Positionierung gegenüber den Universitäten dargestellt. Abschließend werden die Befunde im Kontext aktueller hochschulpolitischer Debatten reflektiert und es werden Schlussfolgerungen für die Governance von Fachhochschulen vorgestellt.

\section{DATEN UND METHODEN}

Die Analyse der Forschungs- und Entwicklungs (FGE) -Aktivitäten im Fachhochschulsektor basiert auf Daten der FH BIS Verordnung, der Österreichischen Forschungsförderungsgesellschaft mbH (FFG), dem Fonds zur Förderung der wissenschaftlichen Forschung (FWF), der Christian Doppler Forschungsgesellschaft (CDG) sowie der Europäischen Kommission und strukturiert diese für unterschiedliche Auswertungen. 
Grundlage für die Identifikation der Forschungsschwerpunkte gemäß der österreichischen Systematik der Wissenschaftszweige (ÖFOS) ${ }^{5}$ sowie für die Analyse der Finanzierung von F\&E-Aktivitäten nach Sektoren bildeten die Daten der FH BIS Verordnung, die auf Informationen basiert, die die Erhalter der Fachhochschulen seit 2012 regelmäßig bereitstellen. ${ }^{6}$

Für eine detailliertere Darstellung der F\&E-Projektfinanzierung durch den öffentlichen Sektor wurden F\&E-Projektbeteiligungen von Fachhochschulen an nationalen und europäischen Forschungsprogrammen aufbereitet, die teilweise öffentlich zugänglich sind (etwa bei Projekten des FWF und der EU-Rahmenprogramme (EU-RP)) bzW. von den jeweiligen Förderagenturen zu Verfügung gestellt wurden (FFG und CDG, relevant für die Josef-Ressel-Zentren (JRZ)). Ü Über die Beteiligung an FqE-Projekten erfolgte auch die Identifikation bestehender Kooperationen mit Universitäten am selben Standort. Des Weiteren wurden Daten zu den Erlösen aus FqE-Projekten aus der Wissensbilanz aufbereitet, die jährlich nach Art des Auftraggebers und Wissenschaftszweig erfasst werden. Im Detail wurden in einem ersten Schritt die Daten zu FGE-Aktivitäten der Fachhochschulen für die Jahre 2014-2016 von den verschiedenen Anbietern gesammelt, Schreibweisen vereinheitlicht und in eine Datenbank integriert. FFG und EU-RP Projekte wurden nach Projekttitel und Laufzeit in den FH BIS Daten identifiziert und fehlende ÖFOS Wissenschaftszweige ergänzt.
Für die derart aufbereiteten Daten wurden deskriptive statistische Analysen durchgeführt. Des Weiteren erfolgte die Analyse der relativen Spezialisierung von Fachhochschulen mittels Revealed Technology Analysis (RTA), ein Instrumentarium zur Erfassung von relativen technologischen Stärken und Schwächen. ${ }^{8}$ Die Berechnung des Kooperationsnetzwerks basiert auf der gemeinsamen Beteiligung an nationalen und europäischen Forschungsprojekten (FFG, FWF und EU-RP).

\section{FINANZIERUNG VON FGE AN FACHHOCHSCHULEN}

Die aktuelle Analyse der Finanzierungsvolumina zeigt einmal mehr die hohe Konzentration der FqE-Aktivitäten an österreichischen Fachhochschulen. Die FH $00 ̈$ und FH Joanneum stellen auf Basis der FH BIS Daten die beiden forschungsstärksten Fachhochschulen dar und decken, gemessen am Gesamtfinanzierungsvolumen, zusammen 45\% von insgesamt 124,5 Mio. EUR für den Zeitraum von 2014 bis 2016 ab. Gleichwohl ist hier zu berücksichtigen, dass die FH 00 und FH Joanneum als die beiden größten Fachhochschulen jeweils an einigen Standorten mit spezialisierten Fachhochschulen aktiv sind. Forschungsaktive Fachhochschulen sind weiters Technikum Wien, FH Kärnten und FH Salzburg, 66\% des Gesamtfinanzierungsvolumens entfallen auf diese fünf forschungsaktivsten Fachhochschulen. Auf die übrigen Fachhochschulen entfallen jeweils weniger als $5 \%$ der Gesamtfinanzierung (Abbildung 1). ${ }^{9}$

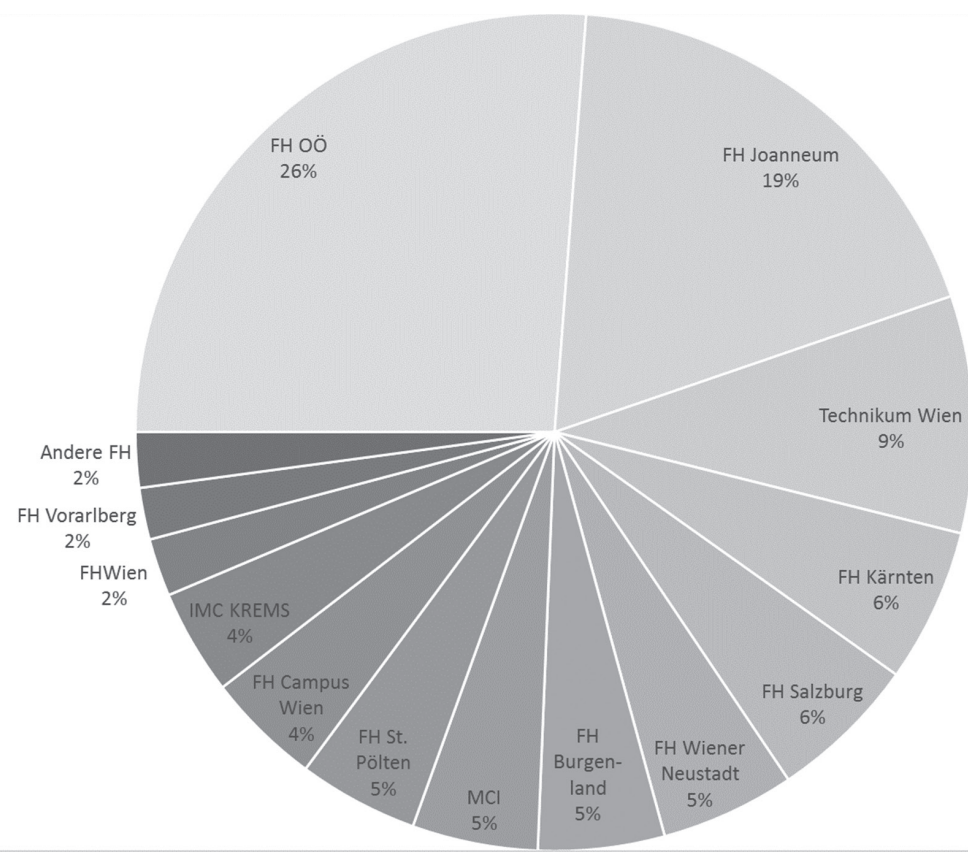

Abbildung 1: Verteilung des Finanzierungsvolumens von FGE (gesamt) Quelle: FH BIS Daten (gesamte externe F\&E-Finanzierung); Berechnung AIT

5 Österreichische Systematik der Wissenschaftszweige 2012 (ÖFOS 2012) ist die auf die österreichischen Gegebenheiten abgestimmte Version der revidierten internationalen Klassifikation ,Fields of Science and Technology' (FOS).

$6 \quad$ Verordnung des Board der Agentur für Qualitätssicherung und Akkreditierung Austria über die Bereitstellung von Informationen über den Studienbetrieb (FH BIS Verordnung, Version 30.7.2012)

7 Dabei hat sich aber auch gezeigt, dass die Finanzierung durch den öffentlichen Sektor nur zum Teil über nationale und europäische Projektbeteiligungen erklärt werden kann und hier deutliche Unterschiede zwischen den einzelnen Fachhochschulen vorliegen. Möglichweise ist bei jenen Fachhochschulen mit geringem Anteil von drittmittelfinanzierten F\&E-Projekten der Anteil der Finanzierung der F\&E über Landes- und Gemeindemittel höher.

$8 \quad$ Vgl. etwa Le Bas und Sierra (2002).

$9 \quad$ Die Reihung der forschungsaktivsten Fachhochschulen entspricht weitgehend der Darstellung in Geyer und Warta (2015) nach Barwert der Förderungen für Projekte der Fachhochschulen bei der FFG und CDG (Tabelle 1, S. 10), wobei FH Vorarlberg und FH St. Pölten in dieser Darstellung noch vor FH Salzburg rangieren. Es scheint, dass beide Fachhochschulen bei der FH BIS Erhebung weniger Finanzierungsvolumen angegeben haben, denn bei Betrachtung des Finanzierungsvolumens über Mittel der FFG und der CDG sind beide Fachhochschulen unter den sieben forschungsaktiven Fachhochschulen. 
Die einzelnen Fachhochschulen unterscheiden sich deutlich in Hinblick auf die Finanzierungsart (Abbildung 2). F\&E an Fachhochschulen wird zu 58\% über den inländischen öffentlichen Sektor finanziert, zu 18\% aus dem Unternehmenssektor und zu 12\% über die EU. Sonstige inländische (andere Fachhochschulen, Universitäten, privater gemeinnütziger Sektor) und ausländische (z.B. Unternehmen) Finanzierungsquellen spielen insgesamt eine untergeordnete Rolle. Einen überdurchschnittlich hohen Anteil an Finanzierung über den öffentlichen Sektor weisen z.B. Technikum Wien (91\%), FH St. Pölten (77\%) und MCI (75\%) auf. Die beiden Fachhochschulen mit dem höchsten Anteil an Finanzierung von F\&E über den Unternehmenssektor sind IMC Krems (56\%) und FH Joanneum (30\%). Bei FH Campus Wien ist der Anteil der Finanzierung über sonstige Inland und hier insbesondere über den gemeinnützigen Sektor auffal- lend hoch (38\%). FH Burgenland zeigt einen überdurchschnittlich hohen Anteil an EU-Mitteln (34\%) und bei der FH Wiener Neustadt ist der Anteil aus dem übrigen Ausland (ohne EU) auffallend hoch (55\%).

Deutliche Unterschiede zeigen sich auch in Bezug auf die Anteile der jeweiligen Fördergeber (FFG, FWF, CDG/JRZ, EU-RP) bei den einzelnen Fachhochschulen (Abbildung 3). Insgesamt sind 57\% der Drittmittel, die von einem der vier Fördergeber stammen, der FFG zuzurechnen, 19\% den JRZ (10\% öffentliche Förderung, 9\% sonstige Förderung), 16\% den EURP und 8\% dem FWF. Überdurchschnittlich hohe Anteile an FFG Mitteln weist FH Joanneum (87\%) auf, während der Anteil der EU-RP Mittel bei der FH Burgenland (26\%) und der FH 00 Ö $23 \%)$ deutlich höher ist. Die beiden Fachhochschulen mit den höchsten Finanzierungsanteilen über die JRZ sind FH Vorarlberg und FH Kärnten (jeweils ca. 50\%).

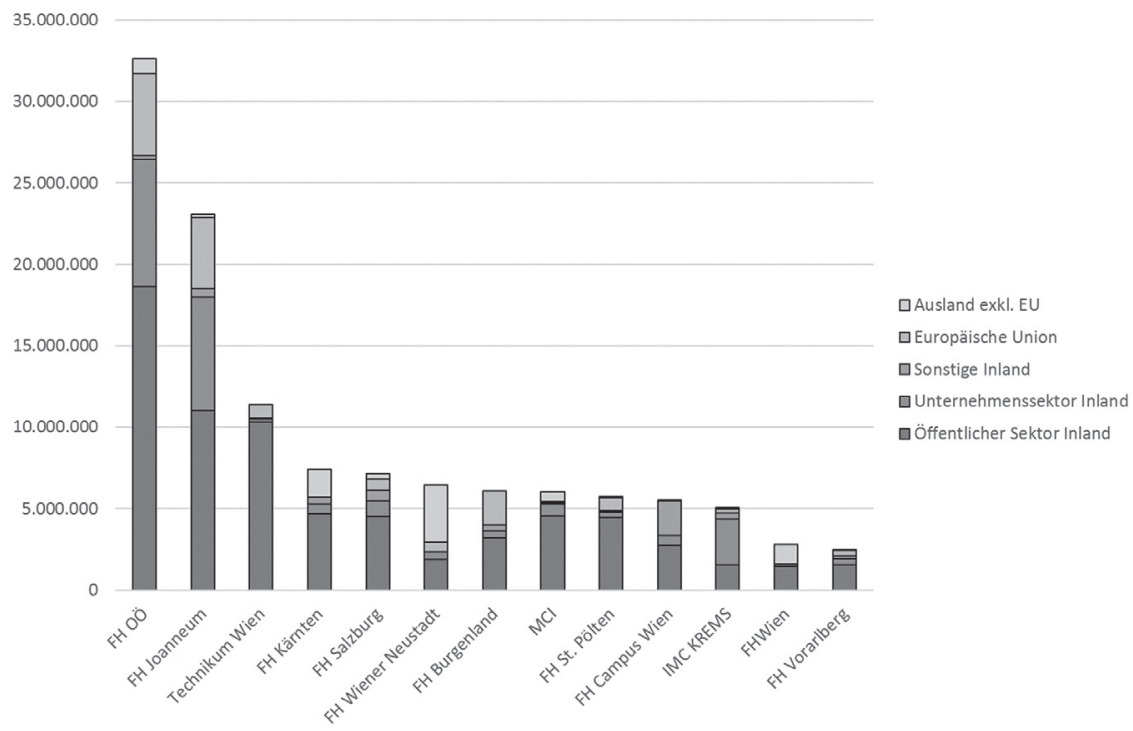

Abbildung 2: Art der Finanzierung von FGE an Fachhochschulen

Quelle: FH BIS Daten (gesamte externe F\&E-Finanzierung); Berechnung AIT

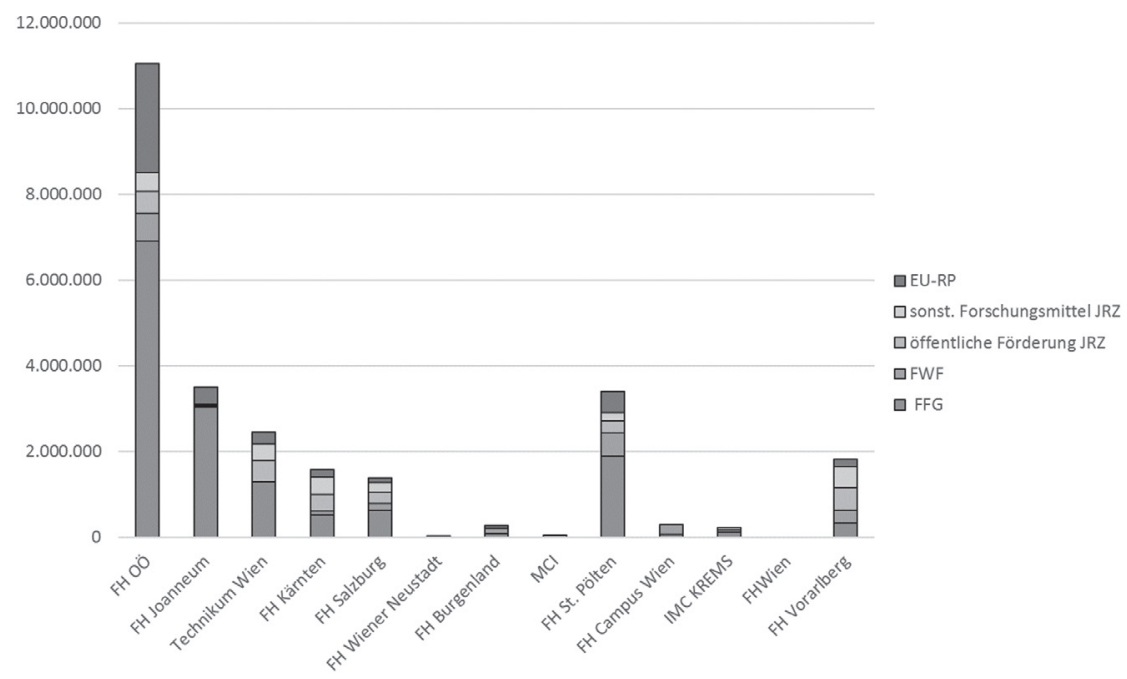

Abbildung 3: Art der Drittmittel-Finanzierung von F\&E an ausgewählten Fachhochschulen

Quelle: FH Drittmittel nach Fördergeber (FFG, FWF, CDG/JRZ, EU-RP); Berechnung AIT 
Die Analyse der Forschungsschwerpunkte über alle Fachhochschulen hinweg zeigt, dass diese in erster Linie in den Technischen Wissenschaften (47\%; insbes. Elektrotechnik, Maschinenbau und Bauwesen) und in den Naturwissenschaften (21\%; insbes. Informatik; Abbildung 4) liegen. Wirtschaftswissenschaften machen $12 \%$ aus gefolgt von Humanmedizin und Gesundheitswissenschaften.

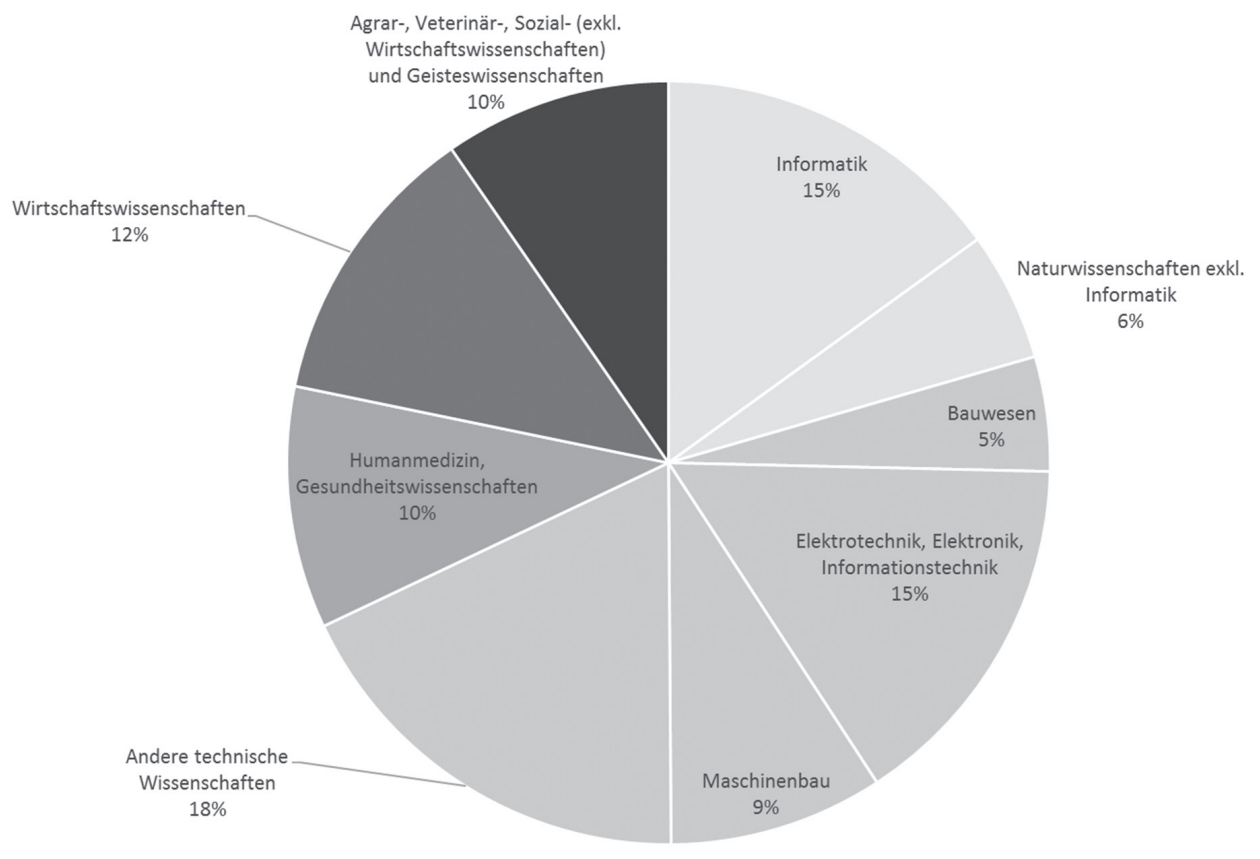

Abbildung 4: Verteilung der thematischen Ausrichtung von F\&E

Quelle: FH BIS Daten (gesamte externe F\&E-Finanzierung); Berechnung AIT

Anmerkung: Andere technische Wissenschaften beinhaltet ÖFÖS 204 bis 211 (Chemische Verfahrenstechnik, Werkstofftechnik, Medizintechnik Umweltingenieurwesen, Angewandte Geowiss., Umweltbiotechnologie, Industrielle Biotechnologie, Nanotechnologie und Andere Technische Wissenschaften), Agrar-, Veterinär, Sozial- (exkl. Wirtschaftswissenschaften) und Geisteswissenschaften beinhaltet ÖFOS 4 bis 6 exkl. 502.

\section{SPEZIALISIERUNG DER FACHHOCHSCHULEN}

Auch in Hinblick auf die thematische Ausrichtung der FGE sind die Fachhochschulen sehr heterogen (Abbildung 5). Dabei ist - wie oben angeführt - jedoch auch zu berücksichtigen, dass die größeren Fachhochschulen teilweise an mehreren Standorten mit einem spezialisierten Angebot tätig sind. Diese Auswertung zeigt etwa einen hohen Anteil an
F\&E von FH Campus Wien und IMC Krems im Bereich Humanmedizin bzw. Gesundheitswissenschaften. Die FH St. Pölten hingegen weist häufig FGE-Aktivitäten in Informatik auf.

10 Der RTA-Index der Spezialisierung der Fachhochschule ist definiert als das Verhältnis des Anteils der FqE-Finanzierung an einer Fachhochschule in einem Wissenschaftszweig an der gesamten FGE-Finanzierung dieses Wissenschaftszweigs und des Anteils der Fachhochschule an der FGE-Finanzierung des gesamten Fachhochschulsektors. Der Wertebereich des Index variiert um 1; wenn für eine Fachhochschule ein Wert größer als 1 errechnet wird, dann liegt eine relative Stärke bzw. Spezialisierung im Vergleich zum gesamten Fachhochschulsektor vor, hingegen liegt bei einem Wert von kleiner 1 eine relative Schwäche der Fachhochschule in der betrachteten Wissenschaftsdisziplin vor. 


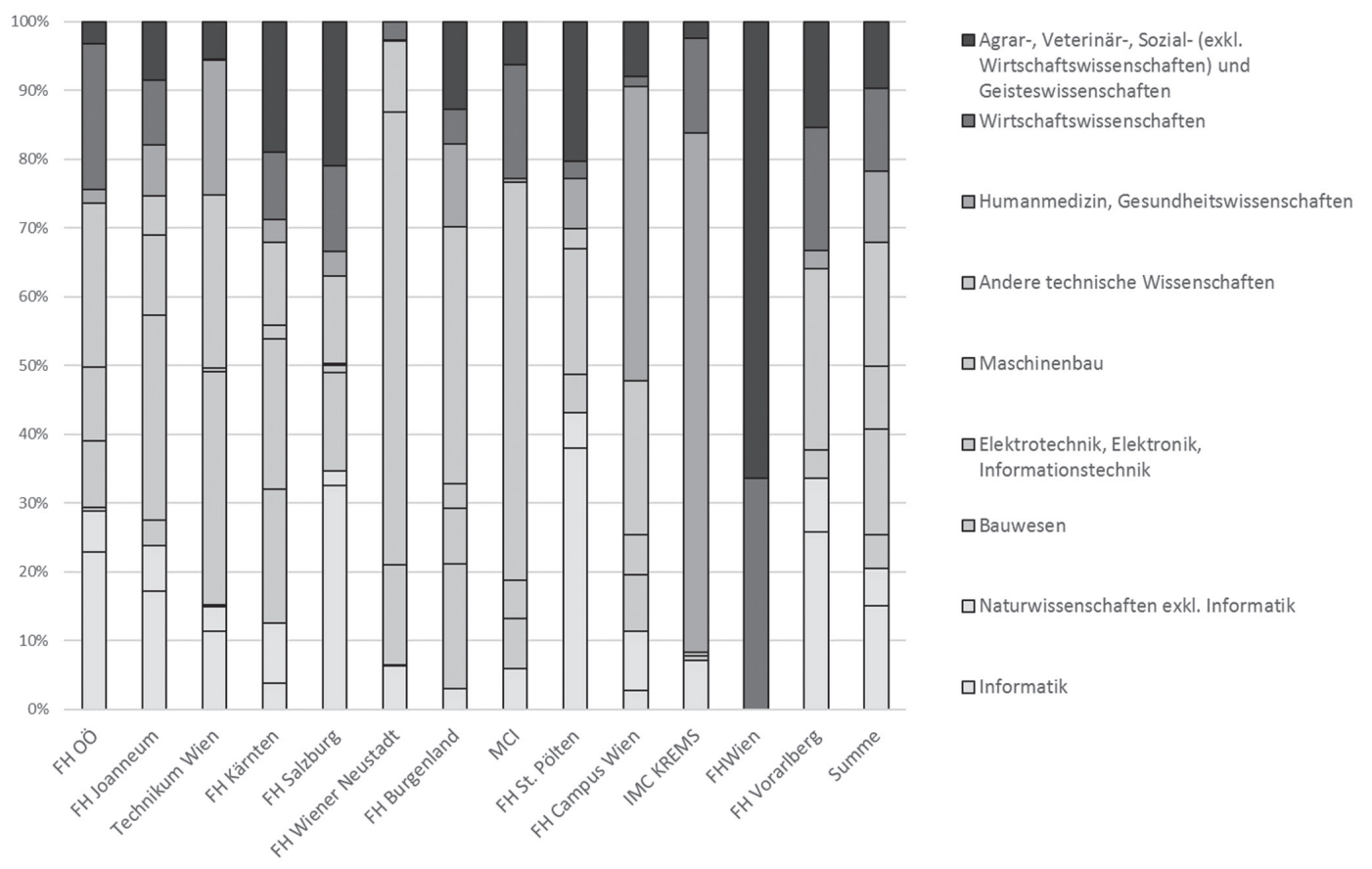

Abbildung 5: Thematische Ausrichtung von F\&E an Fachhochschulen (relativ) Quelle: FH BIS Daten (gesamte externe F\&E-Finanzierung); Berechnung AIT

Neben der prozentuellen Aufteilung der FqE-Mittel nach Wissenschaftszweig kann zusätzlich die relative Spezialisierung mit Hilfe einer Revealed Technology Advantage (RTA) Analyse diagnostiziert werden. ${ }^{10}$ Dabei zeigt die Analyse der forschungsstärksten Fachhochschulen im Vergleich zu allen anderen Fachhochschulen eine relativ breite thematische Ausrichtung mit moderater relativer Spezialisierung (Tabelle 1). Unter den forschungsstärksten Fachhochschulen hat die FH Kärnten eine vergleichsweise starke Fokussierung im Bereich Bauwesen. Die weniger forschungsaktiven Fachhochschulen zeigen insgesamt eine höhere Spezialisierung in einzelnen Themen im Vergleich zum gesamten Fachhochschulsektor. Eine besonders starke thematische Spezialisierung weist etwa die FH Wiener Neustadt im Maschinenbau oder die IMC Krems in der Humanmedizin und den Gesundheitswissenschaften. Die FH Wien zeigt indes eine hohe Spezialisierung im Bereich Sozial- und Geisteswissenschaften.

\begin{tabular}{|l|l|l|l|l|l|l|l|l|}
\hline & Informatik & $\begin{array}{l}\text { Naturwissensch. } \\
\text { exkl. Informatik }\end{array}$ & Bauwesen & $\begin{array}{l}\text { Elektrotechik, } \\
\text { Elektronik, } \\
\text { Informationstechnik }\end{array}$ & Maschinenbau & $\begin{array}{l}\text { Andere } \\
\text { technische } \\
\text { Wissenschaften }\end{array}$ & $\begin{array}{l}\text { Humanmedizin, } \\
\text { Gesundheitswiss. }\end{array}$ & $\begin{array}{l}\text { Wirtschafts- } \\
\text { wissenschaften }\end{array}$ \\
\hline FH 00̈ & 1,52 & 1,09 & 0,11 & 0,62 & 1,19 & 1,31 & 0,20 & 1,75 \\
\hline FH Joanneum & 1,14 & 1,23 & 0,75 & 1,93 & 1,28 & 0,31 & 0,73 & 0,77 \\
\hline Technikum Wien & 0,76 & 0,66 & 0,03 & 2,20 & 0,06 & 1,40 & 1,90 & 0,01 \\
\hline FH Kärnten & 0,26 & 1,59 & 4,02 & 1,42 & 0,22 & 0,66 & 0,32 & 0,81 \\
\hline FH Salzburg & 2,16 & 0,39 & 2,96 & 0,06 & 0,03 & 0,71 & 0,34 & 1,03 \\
\hline FH Wr. Neustadt & 0,00 & 1,17 & 0,02 & 0,94 & 7,25 & 0,57 & 0,01 & 0,22 \\
\hline FH Burgenland & 0,20 & 0,00 & 3,74 & 0,52 & 0,40 & 2,07 & 1,18 & 0,41 \\
\hline MCI & 0,00 & 1,09 & 0,00 & 0,47 & 0,61 & 3,20 & 0,05 & 1,36 \\
\hline FH St. Pölten & 2,52 & 0,94 & 1,15 & 1,18 & 0,00 & 0,16 & 0,72 & 0,20 \\
\hline FH Campus Wien & 0,19 & 1,57 & 1,68 & 0,38 & 0,00 & 1,24 & 4,17 & 0,12 \\
\hline IMC KREMS & 0,00 & 1,29 & 0,00 & 0,05 & 0,00 & 0,03 & 7,37 & 1,13 \\
\hline FHWien & 0,00 & 0,00 & 0,00 & 0,00 & 0,00 & 0,00 & 0,00 & 2,77 \\
\hline FH Vorarlberg & 1,72 & 1,43 & 0,00 & 0,26 & 0,00 & 1,46 & 0,25 & 1,47 \\
\hline
\end{tabular}

Tabelle 1: Relative Spezialisierung der forschungsstärksten Fachhochschulen

Quelle: FH-BIS Daten (gesamte externe FgE-Finanzierung); Berechnung AIT

Anmerkung: Ein Wert von über 1 stellt eine relative Spezialisierung dar, d.h. der Anteil des Wissenschaftszweigs an der jeweiligen Fachhochschule ist größer wie an allen Fachhochschulen. 


\section{POSITIONIERUNG DER}

\section{FACHHOCHSCHULEN IM VERGLEICH ZU UNIVERSITÄTEN}

Fachhochschulen mussten sich seit Beginn ihrer Gründung gegenüber anderen Forschungsakteuren und insbesondere Universitäten positionieren und gehen dabei vielfältige Kooperationen mit Universitäten ein. Dabei stellt sich vor allem die Frage, welche Rolle die räumliche Nähe spielt und in welchen Wissenschaftszweigen kooperiert wird.

Für die Analyse der Kooperation zwischen Universitäten und Fachhochschulen können kooperative FGE-Projekte herangezogen werden. Die Berechnung des Kooperationsnetzwerks basiert auf der gemeinsamen Beteiligung an nationalen und europäischen Forschungsprojekten (FFG, FWF und EU-RP). Die Knoten stellen Fachhochschulen und Uni- versitäten dar, die miteinander verbunden sind, wenn sie in mindestens einem F\&E-Projekt zusammenarbeiten. Je häufiger sie gemeinsam an verschiedenen Projekten beteiligt sind, umso stärker ist ihre Verbindung. Die Größe der Knoten repräsentiert die Anzahl der unterschiedlichen Kooperationspartner; über die Farbe der Knoten sind die Fachhochschulen und Universitäten den Bundesländern zugeordnet.

Die Daten zeigen, dass Fachhochschulen in mehreren F\&E-Projekten mit anderen Fachhochschulen und Universitäten kooperieren und dabei sowohl die geographische Nähe (z.B. FH Joanneum) als auch die thematische Ausrichtung (z.B. FH 0Ö, FH Campus Wien) der Institutionen eine wichtige Rolle spielen (Abbildung 8). Fachhochschulen mit einem vielfältigen Kooperationsnetzwerk sind die FH 00̈, FH Vorarlberg, FH Joanneum und FH St. Pölten. ${ }^{11}$ Forschungsbezogene Kooperationen zwischen forschungsaktiven Fachhochschulen und Universitäten am selben Standort bestehen vor allem in Oberösterreich, der Steiermark und Salzburg.

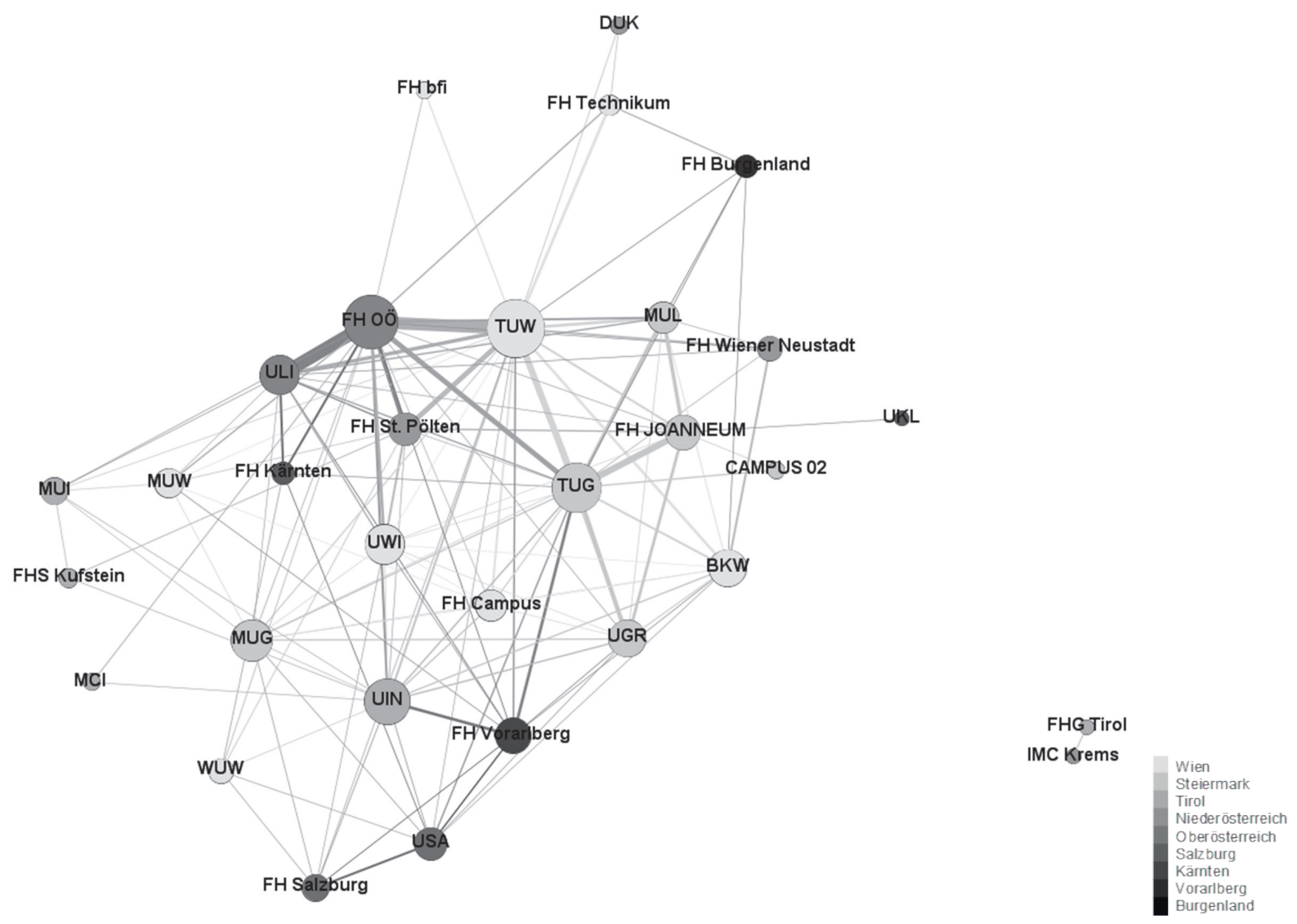

Abbildung 6: Kooperationen zwischen Fachhochschulen und Universitäten

Quelle: FH Drittmittel nach Fördergeber (FFG, FWF, CDG/JRZ, EU-RP); Berechnung AIT

Anmerkung: Universität Wien (UWI), Universität Graz (UGR), Universität Innsbruck (UIN), Medizinische Universität Wien (MUW), Medizinische Universität Graz (MUG), Medizinische Universität Innsbruck (MUI), Universität Salzburg (USA), Technische Universität Wien (TUW), Technische Universität Graz (TUG), Montanuniversität Leoben (MUL), Universität für Bodenkultur Wien (BKW), Wirtschaftsuniversität Wien (WUW), Universität Linz (ULI), Universität Klagenfurt (UKL), Donau-Universität Krems (DUK) 
Für die größte und forschungsstärkste Fachhochschule, die FH OÖ soll hier exemplarisch eine detaillierte Analyse der Spezialisierung der FH 00 vorgestellt werden. Abbildung 7 zeigt für die FH $00 ̈$ eine moderate Spezialisierung in Relation zum gesamten Fachhochschulsektor und im Vergleich zur universitären Forschung eine deutliche Spezialisierung in den technischen Wissenschaften (insbesondere der Elektrotechnik und dem Maschinenbau), der Informatik sowie den Wirtschaftswissenschaften. Diese Spezialisierungen teilt die FH $00 ̈$ mit der Universität
Linz, wodurch ein Kooperationspotential am Standort gegeben ist. Das Gesamtfinanzierungsvolumen für Informatik an der $\mathrm{FH} 00$ beträgt 7,5 Mio. EUR und an der Universität Linz 15,2 Mio. EUR. Wichtige bestehende Kooperationspartner der FH 00 sind neben der Universität Linz am selben Standort insbesondere die beiden technischen Universitäten in Wien und Graz sowie die FH St. Pölten, wie die Berechnung eines EgoNetzwerkes illustriert, hier aber nicht näher dargestellt ist. ${ }^{12}$

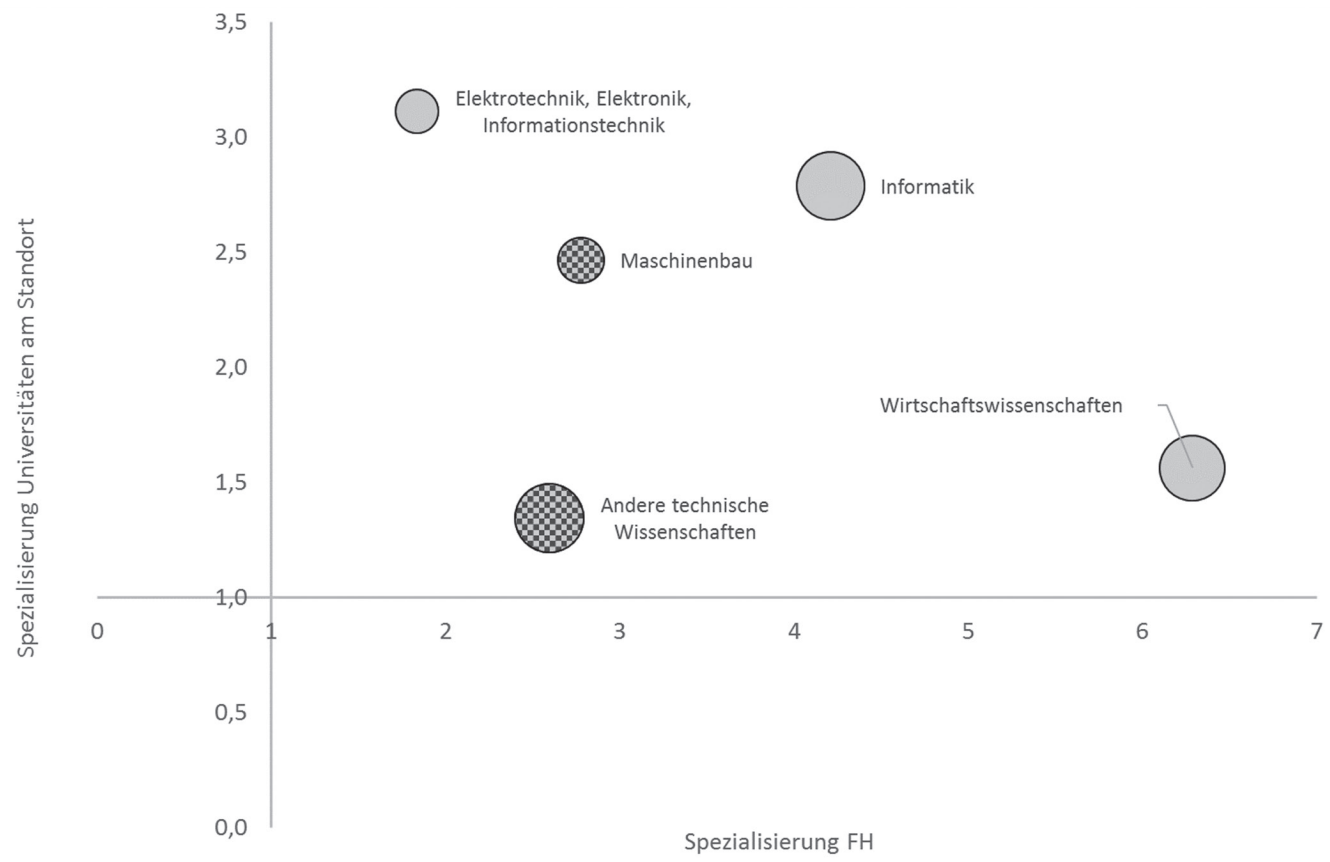

Abbildung 7: Spezialisierungsportfolio FH OÖ und Universität Linz

Quelle: FH BIS Daten (gesamte externe FGE-Finanzierung); Wissensbilanz (Kennzahl 1.C.1.); Berechnung AIT

Anmerkung: Die Größe der Blase stellt die absolute Bedeutung des Forschungsschwerpunkts gemessen am Finanzierungsvolumen an der Fachhochschule dar, gemusterte Blasen bezeichnen Spezialisierungen der Fachhochschule mit einer Infrastrukturinvestition von über 100.000 EUR im Zeitraum 2014 bis 2016; Indikatorwerte über 1 stellen eine relative Spezialisierung dar - die Wissenschaftsdisziplin ist für die jeweilige Fachhochschule bzw. Universität von überdurchschnittlicher relativer Bedeutung.

\section{ZUSAMMENFASSUNG UND DISKUSSION}

Der Fachhochschulsektor ist - gemessen an anderen Forschungseinrichtungen wie der Österreichischen Akademie der Wissenschaften, der Ludwig Boltzmann Gesellschaft und den Universitäten - ein nach wie vor junger Sektor, dem eher geringe Mittel für Forschung zur Verfügung stehen. Der Sektor ist geprägt durch viele Träger mit unterschiedlichen Interessen, was sich nicht zuletzt an den vielen Standorten dokumentiert. Das Hauptinteresse liegt in der Lehre, die Forschung wird durch die größtenteils als Träger auftretenden Länder und das unternehmerische Umfeld geprägt.
Der Fachhochschulsektor konnte seine Forschungsaktivitäten in den letzten Jahren stark ausbauen und einigen Fachhochschulen ist es gelungen, sehr erfolgreich sowohl auf nationaler als auch europäischer Ebene Forschungsmittel im Wettbewerb zu lukrieren. Die öffentliche Hand fungiert als wichtigster Finanzgeber der Forschung. F\&E an Fachhochschulen wird zu 58\% über den inländischen öffentlichen Sektor finanziert und zu 12\% über die EU. Mit 18\% ist aber auch der Anteil der F\&E-Finanzierung aus dem Unternehmenssektor an Fachhochschu- 
len größer als an den Universitäten. Weitere inländische (z.B. privater gemeinnütziger Sektor) und ausländische Finanzierungsquellen (z.B. Unternehmen) spielen eine untergeordnete Rolle.

Auf Basis der Analyse der thematischen Ausrichtung der Forschungsaktivitäten zeigt sich der Fachhochschulsektor als sehr heterogen: Die forschungsstärksten und zugleich größeren Fachhochschulen weisen dabei eine relativ breite thematische Ausrichtung mit moderater relativer Spezialisierung gegenüber dem gesamten Fachhochschulsektor in mehreren Themen auf. Die weniger forschungsaktiven Fachhochschulen weisen hingegen meist eine höhere Spezialisierung in einzelnen Themen auf.

Natürlich beeinflussen auch die Wissenschaftsdisziplinen selbst die Art der Finanzierung maßgeblich. So zeigt sich etwa, dass in manchen Wissenschaftsdisziplinen auch Fachhochschulen in der Lage sind FWF-Mittel - also Grundlagenforschungsmittel - (z.B. Informatik oder Humanmedizin) zu lukrieren, während in anderen FFG-Mittel eine überproportional große Bedeutung haben (z.B. Maschinenbau). Die Finanzierungsstruktur der einzelnen Fachhochschulen ist demzufolge auch abhängig von deren Spezialisierung. Im Vergleich zur Finanzierung der FqE an Universitäten in gemeinsamen Schwerpunkten lässt sich als gemeinsamer Trend über alle Standorte und Wissenschaftsdisziplinen hinweg nur die insgesamt größere Bedeutung von FWF-Mitteln für die Universitäten feststellen. Im Gegensatz dazu sind Fachhochschulen am Standort teilweise zu einem größeren Anteil über Mittel der FFG oder aus dem Unternehmenssektor finanziert. Dieser Befund zeigt einmal mehr, dass eine strikte institutionelle Trennung zwischen Grundlagenforschung und angewandter Forschung nicht mehr aufrechterhalten werden kann.

Bei der Gegenüberstellung der Spezialisierung von Fachhochschulen und Universitäten am selben Standort setzt sich das heterogene Bild fort. So zeigen sich in Oberösterreich gemeinsame Spezialisierungen der FH 00 und der Universität Linz in einer Reihe von Wissenschaftszweigen, wie z.B. Elektrotechnik, Maschinenbau, Informatik. An den großen Universitätsstandorten Wien und Graz (bzw. Steiermark), die auf Seiten der Universitäten sehr breit diversifizierte Forschungsportfolios aufweisen, haben Fachhochschulen und Universitäten nur in einzelnen Bereichen gemeinsame Spezialisierungen (z.B. Maschinenbau in Graz). Weiters führen die Fachhochschulen Forschung in Bereichen durch, die nicht von Universitäten am Standort abgedeckt werden (z.B. Bauwesen in Kärnten und Salzburg). Teilweise gelingt es Fachhochschulen auch Nischen zu besetzten, die österreichweit einzigartig sind, wie etwas das Beispiel der Aviation der FH Joanneum illustriert.

F\&E-Kooperationen zwischen Fachhochschulen und Universitäten am selben Standort bestehen vor allem in Oberösterreich, der Steiermark und Salzburg. Darüber hinaus sind überregionale Kooperationen mit Universitäten mit ähnlichem Spezialisierungsportfolio zu beobachten (z.B. bei FH Kärnten und FH St. Pölten). Wiener Fachhochschulen kooperieren in FGE-Projekten - trotz vorhandener Kooperationspartner - vergleichsweise selten mit Universitäten am Standort. Möglicherweise besteht hier eine engere Bindung zur außeruniversitären Forschung, wie etwa das Beispiel FH Technikum Wien und Austrian Institute of Technology illustriert. Es mag aber auch sein, dass die Wiener Fachhochschulen, die in einem sehr dichten universitären Umfeld agieren und weniger indus- trielle Partner im Vergleich zu Fachhochschulen in Bundesländern vorfinden, einen tendenziell stärkeren Fokus auf den Bereich Lehre legen.

Die thematische Ausrichtung und Spezialisierung der Fachhochschulen ist ebenso vom regionalen und nationalen Umfeld geprägt, wie sie das Ergebnis von organisatorischen Strategien ist. Ein Teil der österreichischen Fachhochschulen hat unter Einbeziehung der Interessen unterschiedlicher Stakeholder in der Vergangenheit Forschungsstrategien entwickelt. In einigen Bundesländern erfolgt dies auch in enger Abstimmung mit der Landespolitik, die ihrerseits ebenfalls die Fachhochschulen finanziell unterstützt. Die Strategiebildung erfolgt in der Vergangenheit dabei auch unter Bezugnahme auf die Philosophie der Smart Specialisation. ${ }^{13}$ Gleichzeitig sind die Fachhochschulen bei der Entwicklung ihrer Forschungsstrategien an ihre Mission gebunden. Nach dem Hochschulentwicklungsplan stellt die Kernaufgabe der Fachhochschulen die Lehre dar, weshalb die Forschung zum größten Teil in ihren Schwerpunktsetzungen von der Lehre getrieben wird. Dies bedeutet auch bei der Aufnahme von neuem Personal eine gewisse Schwerpunktsetzung in der Lehre und keine Ausrichtung als reine "Forschungsprofessur".

Während Fachhochschulen wie auch andere Akteure des österreichischen Hochschulsystems (Universitäten, Fachhochschulen und Privatuniversitäten) sich voneinander unabhängig eher "naturwüchsig” entwickelt haben, wäre nunmehr ein akkordiertes Vorgehen zwischen den unterschiedlichen Trägern in der Abstimmung der Forschung empfehlenswert. So wären bereits vorhandene kooperative Ansätze - etwa in der Doktoratsausbildung - weiterzuentwickeln und würde es beispielsweise den Fachhochschulen erlauben, zusätzliches „Forschungspersonal" aufbauen zu können. Gleichzeitig könnten diese Kooperationen zur Intensivierung des Austauschs mit der Wirtschaft beitragen und eine Innovationskette von der Grundlagenforschung bis zum Produkt an Personen festmachen. Durch eine enge regionale Vernetzung und Interaktion können wichtige Impulse für die Entwicklung von neuen, innovativen Produkten, Dienstleistungen und Prozessen gegeben und damit Wettbewerbsvorteile für alle beteiligten Organisationen entstehen. ${ }^{14}$ Dabei ist jedoch, wie oben angeführt, zu berücksichtigen, dass das Agenda Setting in den Fachhochschulen überwiegend lokal geprägt (Unternehmen, Länder) ist und sich vielfach einer stärkeren nationalen Koordinierung entzieht. Zudem sind die Finanzierungsspielräume der Fachhochschulen aufgrund des hohen Anteils kompetitiv eingeworbener Mittel eingeschränkt.

Die Sicherstellung regional ausgewogener Angebotsstrukturen in der Lehre und damit verbundene Ressourcenallokation würde eine abgestimmte Gesamtplanung ebenfalls als sinnvoll erscheinen lassen und die Frage aufwerfen: welche Fächer sollen wo angeboten werden? Dabei können regionale Überlegungen aus der Lehre, z.B. Tourismus im steirischen Kurort Bad Gleichenberg, genauso von Bedeutung sein wie Personalbedarf und Forschung im Nahebereich von Industrie wie in Linz. Forschungspolitik würde hier teilweise als Strukturpolitik eingesetzt, weshalb Standortfragen im Spannungsfeld von Regionalpolitik vs. Wissenschaftspolitik in diesem Abstimmungsprozess eine wesentliche Rolle spielen. In der Abstimmung müssten aber auch andere regionale Forschungseinrichtungen mit berücksichtigt werden. Daraus könnten sich auch neue Themenbereiche für die Lehre ergeben. Potentielle neue

\footnotetext{
13 Vgl. http://wissenschaft.bmwfw.gv.at/bmwfw/forschung/national/standortpolitik-fuer-wissenschaft-forschung/ris3-standortstrategien-fuer-smart-specialisation/

$14 \quad$ Vgl. Berlin-Partner (2015).
} 
Forschungskooperationen könnten sich für Fachhochschulen entweder auf Basis gemeinsamer Spezialisierungen mit anderen Forschungseinrichtungen am Standort ergeben. Weiters können aber beispielsweise auch komplementäre Spezialisierungen der Fachhochschulen und anderer Forschungseinrichtungen am Standort genutzt werden, um gemeinsam neue transdisziplinäre Forschungsfelder zu etablieren.

Vor dem Hintergrund der Notwendigkeit abgestimmter Prozessabläufe der strategischen Planung zwischen den einzelnen Trägern stellt sich damit die Grundsatzfrage: Wie kann das Wechselspiel zwischen zentraler Planung im Ministerium/Landesverwaltung und dezentralen Planungen in den Hochschulen gestaltet werden und wer ist der strategische Vordenker mit welchem Konkretisierungsgrad? Geht man von einem gesamthaften Hochschulraum aus, dann liegt diese Verantwortung beim Bund, wobei es hier ein Abstimmungsmechanismus zu den Ländern aufgrund von diesen finanzierten „Länderuniversitäten“ (Privatuniversitäten) und Fachhochschulen geben sollte. Eigene Forschungsverbünde könnten die oft nicht ausreichend vorhandenen Ressourcen für Forschung an den Fachhochschulen sinnvoll stärken und durch Clusterungen regional wichtige Forschungsthemen für KMUs oder die Industrie vorantreiben. So ließen sich auch die unterschiedlichen kompetitiven Mittel sinnvoll zusammenfügen.

\section{REFERENZEN}

BMWFW (2015) Fachhochschul-Entwicklungs- und Finanzierungsplan >>2017/18, Wien.

Berlin-Partner (2015): Zukunftstechnologien von der Idee zum innovativen Produkt. Berlin, online http://www.berlin-partner.de/branchencluster/

Burkert, G. R. (2019), „Universitas digitalis - quo vadis?” ZVernetzte Universitäten in neuer gesellschaftlicher Verantwortung. Magazin Erwachsenenbildung.at, Heft 35 (in Druck).

Dinges, M., Leitner, K-H., Zahradnik, G. (2014): Evaluierung des Programmes FHplus, Auftragsprojekt für das BMVIT, AIT-IS-Report, Juni, Wien.

Geyer, A. und Warta, K. (2015): Evaluierung der Forschungsförderung für Fachhochschulen in Österreich. Technopolis, Wien.

Heller-Schuh, B., Zahradnik, G., Leitner, K-H. (2018): Forschung an Fachhochschulen. Analyse forschungsrelevanter Fachhochschuldaten, Auftragsprojekt für das BMBWF, AIT-ISP-Report 14, April, Wien.

Le Bas, C.L. und Sierra, C. (2001): 'Location versus home country advantages' in R\&D activities: Some further results on multinationals' locational strategies. Research Policy 31(4), 589-609

Österreichischer Wissenschaftsrat (2012): Fachhochschulen im österreichischen Hochschulsystem. Analysen, Perspektiven, Empfehlungen, Wien.
Paier, D. (2012): Die Entwicklung der F\&E-Agenda im österreichischen Fachhochschulsektor. Zeitschrift für Hochschulentwicklung, 7, 2, 12-31.

Pichl, E. (2017): Differenzierung im Hochschulsystem. Zwischen Bildungsauftrag und Selbstentwurf, Österreichischer Wissenschaftsrat, Tagungsband.

Steiner, R. et al. (2006): Zwischenevaluierung FHplus, KMU-Forschung Austria und Fraunhofer Institut System- Und Innovationsforschung, Wien.

Stucke, A. (2014): Der Wissenschaftsrat als Verhandlungssystem - und was man daraus für die "Governance" der Wissenschaft lernen kann. In: Rene Kremgkow, Andre Lottmann, Torger Möller (Hg.): Völlig losgelöst? Governance der Wissenschaft. Band der 6.iF0-Jahrestagung.

\section{AUTORiNNEN}

\author{
GÜNTHER R. BURKERT \\ Department für E-Governance \\ Donau-Universität Krems \\ Dr.Karl-Dorrek-Straße 30 \\ 3500 Krems \\ E: Guenther.burkert@donau-uni.ac.at
}

BARBARA HELLER-SCHUH

AlT Austrian Institute of Technology

Center for Innovation Systems \& Policy

Giefinggasse 4, 1210 Wien

E: barbara.heller-schuh@ait.ac.at

\section{KARL-HEINZ LEITNER}

AlT Austrian Institute of Technology

Center for Innovation Systems \& Policy

Giefinggasse 4, 1210 Wien

und

Karl-Franzens-Universität Graz

Zentrum für Entrepreneurship und angewandte Betriebswirtschaftslehre

Elisabethstraße 50b/ll, 8010 Graz

E: karl-heinz.leitner@ait.ac.at

\section{GEORG ZAHRADNIK}

AlT Austrian Institute of Technology

Center for Innovation Systems \& Policy

Giefinggasse 4, 1210 Wien

E: georg.zahradnik@ait.ac.at 